Revue

Revue de l'histoire des religions

de Ihistoire des religions

1 | 2018

Varia

\title{
Bouffons et clowns sacrés de l'Himālaya
}

Sacred Jesters and Clowns in the Himālaya

\section{Gérard Toffin}

\section{Q OpenEdition}

Journals

Édition électronique

URL : http://journals.openedition.org/rhr/8846

DOI : 10.4000/rhr.8846

ISSN : 2105-2573

Éditeur

Armand Colin

Édition imprimée

Date de publication : 1 mars 2018

Pagination : 97-131

ISBN : 978-2-200-93166-7

ISSN : 0035-1423

\section{Référence électronique}

Gérard Toffin, «Bouffons et clowns sacrés de l'Himālaya », Revue de I'histoire des religions [En ligne], 1 | 2018, mis en ligne le 01 mars 2020, consulté le 15 janvier 2021. URL : http://journals.openedition.org/ rhr/8846 ; DOl : https://doi.org/10.4000/rhr.8846

Tous droits réservés 


\section{GÉRARD TOFFIN}

Centre national de la recherche scientifique

\section{Bouffons et clowns sacrés de l'Himālaya}

Les populations des régions himālayennes dissocient mal le comique du sacré. Cinq catégories principales de clowns et de farceurs interviennent dans la vie cérémonielle et les fêtes collectives : les bouffons/faiseurs de tours, les clowns brāhmanes ou ascètes hindous, les clowns atsara tibétains, les bouffons chamaniques et les démons personnifiés. Ces personnages comiques s'apparentent aux puissances surnaturelles. Ce sont des figures équivoques, ambiguës, à la fois inquiétantes et attirantes, qui font sauter en éclats les frontières, renversent les statuts et introduisent un vent de liberté. Elles effrayent les mauvais esprits, plaisent aux divinités et concourent aux rituels de fertilité. Ces représentations appartiennent aux aspects les plus savants du religieux.

\section{Sacred Jesters and Clowns in the Himālaya}

The populations of the Himalayan regions do not neatly dissociate the comic from the sacred. Five main categories of clowns and tricksters take part in ceremonial life and in collective festivals : jesters/magicians, Brahmins or Hindu ascetics, Tibetan atsara clowns, shamanic jesters and personified demons. These comic characters are closely related to supernatural powers. They are equivocal, ambiguous figures, at once frightening and enticing, who overturn roles and stir up the winds of liberty. They frighten away evil spirits, placate divinities and oversee fertility rituals. These representations are among the most erudite aspects of the religious realm. 
«L'esprit de bouffonnerie est le seul qui autorise certaines audaces. »

Jean Cocteau, Les Mariés de la Tour Eiffel,

(Paris, Gallimard, 1926)

Dans La Pensée sauvage, Claude Lévi-Strauss oppose le jeu au rite en ces termes : «Le jeu, affirme-t-il, apparaît (...) comme disjonctif: il aboutit à la création d'un écart différentiel entre des joueurs individuels, ou à des camps, que rien ne désignait au départ comme inégaux. Pourtant, à la fin de la partie, ils se distingueront en gagnants et perdants. De façon symétrique et inverse, le rituel est conjonctif, car il institue une union (on peut dire ici une communion), ou, en tout cas, une relation organique, entre deux groupes (...) qui étaient dissociés au départ (...). Dans le cas du jeu, la symétrie est préordonnée (...). L'asymétrie, elle, est engendrée (...). Dans le cas du rituel on pose une asymétrie préconçue entre profane et sacré, fidèles et officiants, morts et vivants, initiés et non-initiés, etc., et le «jeu» consiste à faire passer tous les participants du côté de la partie gagnante, au moyen d'événements dont la nature et l'ordonnancement ont le caractère véritablement structural $»^{1}$. Le jeu marquerait les différences, le rite l'égalité.

Comme toutes les élaborations théoriques de Lévi-Strauss, ces réflexions sont complexes, et, qui plus est, suspendues comme en apesanteur, sans ancrage bien circonscrit. Rien d'étonnant à ce qu'elles puissent susciter des interprétations pour le moins différentes, voire à ce qu'elles soient totalement rejetées. Elles valent cependant la peine d'être citées en introduction à la présente enquête. En première analyse, la comédie et le rire, qui appartiennent à la sphère du jeu, s'opposent en effet au rite à peu près dans les mêmes termes que ceux employés par Lévi-Strauss. Le rite exclut en principe le rire, c'est même le domaine de l'antirire. Le premier vise à colmater une fissure, à réparer un désordre,

1. Claude Lévi-Strauss, Euvres complètes. Paris, Gallimard, Bibliothèque de la Pléiade (édition de Vincent Debaene), 2010, p. 595. 
à recréer une unité menacée par tel ou tel esprit malfaisant, ou à refonder une entité, une communauté autour de quelques principes communs; il est soumis à de multiples interdits. Le rire (dont les formes sont diverses et dont les liens avec l'humour, la plaisanterie, l'allégresse, le simple sourire méritent d'être spécifiés à chaque fois) induit, lui, fréquemment une dissonance, un décalage, voire un chahut ou un charivari. Il raille, dénigre les atypiques, les comportements jugés asociaux. Il fait souffler un vent de liberté. Il est, ce faisant, dans l'hyperbole et l'exagération, il force le trait.

Le christianisme et, d'une manière générale, les grandes religions monothéistes tendent à bannir le rire de l'espace religieux et cérémoniel. Rit-on durant une messe ou durant une prière ? Ce serait une offense au Christ, aux souffrances qu'il a endurées. Jacques Le Goff nous a rappelé que dans la Chrétienté médiévale, « la source du rire est localisée dans le bas du corps humain, ce qui l'entraîne dans le domaine de la luxure et du diable $»^{2}$. Les règles monastiques le bannissaient ${ }^{3}$. Le Christ est l'homme qui ne rit jamais ; il est l'agélaste par excellence, celui qui ne sait pas rire ${ }^{4}$. L'homme, qu'il a façonné à son image, est tenu de se conformer à ce modèle. Quant à l'islam, les récentes polémiques relatives aux caricatures du Prophète attestent de la difficulté des autorités religieuses à admettre la dérision et la moquerie quand il s'agit de dieu. Le sacré exige d'être protégé de ce qui est perçu comme une profanation. Le rire choque, il porte atteinte à la dignité divine, il irrite les entités surnaturelles. Il ressemble au cri du singe, au hennissement du cheval ou à l'aboiement du chien, il confond les barrières entre l'homme et l'animal. De plus, tout élément comique risque de distraire et perturber le bon déroulement d'opérations qu'il convient d'exécuter le plus minutieusement possible.

Le christianisme a certes admis durant longtemps que ses ouailles se livrent à des parodies du sacré, qu'ils mettent en scène de manière comique ses ministres du culte et ses dogmes les plus fondamentaux à certaines périodes limitées du calendrier religieux.

2. Cf. Jacques Le Goff, Pour un autre Moyen Age. Paris, Gallimard, 1977, p. 461.

3. Jacques Le Goff, «Le rire dans les règles monastiques du haut Moyen Âge », in Un autre Moyen Âge, Paris, Gallimard, 1999, p. 1357-1368.

4. Sur cette question qui est cependant controversée, voir Jacques Le Goff, « Jésus a-t-il ri ? », L'Histoire, 158, 1992, p. 72-74. 
Mais ces manifestations carnavalesques, critiques, furent vite déconsidérées, puis réprimées par les autorités ecclésiastiques qui y virent du paganisme et par les pouvoirs constitués pour qui il y avait là une source de désordre. Le rire subversif, païen fut donc pourchassé. Il trouva refuge dans les domaines extra-religieux et les pratiques populaires. Il y eut scission, disjonction entre sacré et comique.

Qu'en est-il des univers plus lointains où le polythéisme domine ? Prenons l'exemple de l'Himālaya, une région du monde où le bouddhisme tantrique (et, de plus en plus, le Theravāda) côtoie un hindouisme savant et populaire ainsi que des cultes plus anciens et des formes de chamanisme. Dans tous les pays de cette zone (Népal, Inde du Nord, Tibet, Bhoutan), la religiosité est extrême. La religion enveloppe ici l'ensemble de la vie sociale et la vie quotidienne, elle est le pivot des valeurs dominantes. Peu d'espaces lui échappent. Hormis une petite minorité tournée vers l'Occident et déconnectée de ses traditions, les populations de l'Himalaya vivent de prédictions et de présages. Elles croient aux danseurs masqués, aux mythes et aux fêtes du calendrier lunaire. Le comique n'a certes pas besoin de sacré pour s'exprimer, mais les deux versants sont très souvent liés. Aujourd'hui comme hier, rire et comique sont bien présents dans la vie cérémonielle ${ }^{5}$. Ils y ont leur place, une place qui ne se réduit pas seulement à des moments récréatifs. Un sens plus profond, souvent caché aux profanes, s'y fait jour. Le comique, antithèse du sérieux liturgique, à la fois figure de vitalité et d'ambiguïté, acquiert alors une signification religieuse positive. À quelques exceptions près, dont j'aurai à traiter, on n'y voit pas (ou pas seulement) des expressions sataniques, maléfiques, comme dans le christianisme ${ }^{6}$.

Je me propose de traiter cette question et d'analyser les relations entre comique et sacré sur la base de quelques exemples

5. Les représentations connues communément sous le nom de «Bouddha riant » (en chinois Budai ou Pu-Tai), une figure du bouddhisme chinois et des pays sinisés comme le Vietnam, souvent identifié à Maitreya, ne désigne pas en fait le Bouddha, mais un moine qui aurait vécu en Chine au $\mathrm{X}^{\mathrm{e}}$ siècle de notre ère. $\mathrm{Ce}$ personnage divinisé apporte bonheur, abondance et richesse.

6. Contrairement à Lévi-Strauss, Michel Leiris relevait dès 1958 les multiples connexions existant entre le rituel et le jeu dans son étude sur les rituels de possession en Afrique noire. Voir La possession et ses aspects théâtraux chez les Éthiopiens de Gondar, Paris, Plon, 1958. 
ethnographiques himālayens représentatifs. Les sources écrites dans les langues locales de l'Himalaya sont rares, il faut se laisser guider par l'observation, l'écoute et le dialogue. Qu'observet-on alors? Dans de nombreux cas, la règle édictée par LéviStrauss s'applique: le rire, élément perturbateur, est exclu des liturgies et des rituels. Il est source de désordre et peut donc nuire à l'efficacité attendue de la cérémonie. Les démons et mauvais esprits, congénitalement des fauteurs d'obstacles, sont toujours prêts à faire dérailler les rituels qui risquent de leur nuire.

Mais dans de nombreuses autres occasions coutumières le rire est prescrit. C'est le cas des cérémonies festives, des mascarades de rue ou des farces représentées sur une scène de théâtre en plein air, parfois des liturgies sacrées. Toutes ces manifestations à forte connotation religieuse sont composées d'un petit nombre d'éléments fixes (déguisement, travestissement d'homme en femme, autres types d'inversion, danse, mascarades jouées par des personnages de bas statut ou caste, course de garçons après les filles, etc.) qui s'avèrent indispensables à la réussite de l'événement festif. Elles relèvent à la fois du rite et du jeu. Bien qu'elles soient célébrées à jour fixe, le plus souvent selon le calendrier lunaire religieux, elles entrent dans la catégorie de l'anti-structure, subversive, telle que l'a établie Victor Turner. Les hiérarchies du temps profane se noient soudain dans un flot égalisateur et un tumulte.

De telles mascarades jouent par ailleurs un rôle indispensable dans le rite. Elles créent un effet de surprise, elles mettent en scène des figures équivoques, à la fois inquiétantes et attirantes, qui participent à la cérémonie et contribuent à son efficacité? Leur exubérance même parfois effraye. C'est cet autre versant du religieux que je veux aborder ici. Je prendrai appui, entre autres, sur le livre récent de Roberte Hamayon, Jouer ${ }^{8}$, qui insiste sur la présence du jeu dans de très nombreux rituels de par le monde,

7. D. Handelman, "The Ritual-Clown: Attributes and Affinities", Anthropos, 1981, 76 (3-4), p. 321-370.

8. Jouer, Une étude anthropologique, Paris, La Découverte («Bibliothèque du Mauss »), 2012. La tentative de Roberte Hamayon de regrouper dans la notion de jeu un ensemble d'activités fort diverses apparaît cependant peu convaincante. À trop élargir la notion, on risque de la rendre peu pertinente. Les faits rituels ou festifs analysés dans la présente étude ont peu à voir, par exemple, avec les jeux d'échecs ou les Jeux Olympiques. 
et particulièrement en Mongolie et Sibérie. Dans la phrase citée plus haut, Claude Lévi-Strauss semble oublier cet aspect ludique et ambigu du sacré. C'est pourtant là où le comique surgit ${ }^{9}$.

Cinq grands types de personnages comiques se produisent lors des fêtes religieuses en question ${ }^{10}$ : les bouffons/faiseurs de tours (type «trickster»), les clowns brāhmanes ou ascètes hindous, les clowns atsara tibétains, les bouffons chamaniques et les représentations de démons. Certaines figures clownesques échappent peut-être à cette classification, mais leurs liens avec le religieux paraissent plus ténus. Tous les personnages retenus ici, sauf peut-être le dernier, peuvent être qualifiés de bouffons ; tous sont à des degrés divers des clowns. Ils se caractérisent tous par le choc visuel qu'ils provoquent, des métamorphoses, la rupture d'avec l'ordre quotidien, l'excès. Ces figures comiques possèdent cependant chacune des traits particuliers qui tiennent au contexte rituel dans lequel elles s'insèrent et à l'arrière-plan culturel qui les détermine. J'insisterai sur ces contextes qui relèvent souvent de la tradition savante. Ils déjouent les généralisations abusives. Les modèles anthropologiques importent, mais leur niveau d'abstraction empêche parfois le chercheur de saisir les spécificités et les subtilités - cruciales dans une question aussi complexe que le rire.

Un livre récent (2014) de Pascale Dollfus et de Gisèle Krauskopff rassemble sur le sujet un riche corpus ethnographique accompagné d'une iconographie de qualité ${ }^{11}$. Il convient à présent de faire une analyse approfondie de ces festivités et de ces spectacles durant lesquels, comme l'écrit Baudelaire : «le peuple oublie tout, la douleur et le travail $»^{12}$. Pour l'heure cette analyse du comique sacré fait défaut. Nous tenterons de donner

9. Ce texte est paru sous une forme légèrement différente dans La Lettre du Toit du monde, $\mathrm{n}^{\circ} 20$, mars 2017, avec de nombreuses illustrations.

10. Dans certains cas, plutôt rares, les thèmes bouffons ne sont pas incarnés sur scène ; ils interviennent uniquement dans les mythes et la littérature orale. On en rencontrera quelques exemples dans l'analyse.

11. Pascale Dollfus et Gisèle Krauskopff, Mascarades en Himalaya, Les vertus du rire. Suilly-la-Tour, Éditions Findakli, 2014. Voir le compte rendu de Roberte Hamayon, Archives des Sciences Sociales des Religions, $\mathrm{n}^{\circ}$ 172, 2015, p. 292.

12. Charles Baudelaire, «Le Vieux Saltimbanque», Le Spleen de Paris, Euvres complètes, Paris, Gallimard («Bibliothèque de la Pléiade ») (édition Claude Pichois), 1975, I, p. 295. 
une forme plus générale à nos observations sans oublier qu'il s'agit ici d'une étude préliminaire destinée à être complétée et enrichie par des recherches plus poussées sur le sujet. Une précision avant de commencer l'enquête. Au rire, on préférera ici le terme de comique. La première notion paraît en effet plus limitée que la seconde, laquelle a l'avantage d'élargir le questionnement et d'ouvrir le plus d'horizons. Le comique couvre en effet des phénomènes fort variés ; il inclut le rire et le faire rire, le renversement festif et la parodie. Il introduit une dimension supplémentaire qui est celle du genre de la performance théâtrale ou chorégraphique, de ses modalités, là purement gestuelles, ailleurs dialoguées, ici totalement orales, là orales et écrites.

\section{LES BOUFFONS/FAISEURS DE TOURS (TRICKSTERS) HIMĀLAYENS}

La littérature tibétaine est riche en bouffons/faiseurs de tours de toutes sortes. Très souvent, ce type de personnage incarne l'homme du commun excellant à rouler son propriétaire et les féodaux qui le gouvernent. Les tours joués par le ministre Nyichoe Zangpo au roi de Nedhong, un rustre, sont également célèbres dans la mythologie du pays, de même que ceux de Oncle Tompa, un héros du folklore local, qui réussit à vendre des pénis dans une nonnerie et à coucher avec la fille du roi ${ }^{13}$. Un autre fameux trickster tibétain est Drukpa Kunley (1455-1529), un personnage historique qui appartient à la catégorie des « fous divins » dont on reparlera plus $\operatorname{loin}^{14}$.

La figure comique du trickster est également présente dans les traditions orales des populations tribales (parlant des langues tibéto-birmanes) de l'est himālayen, notamment en Assam et en Arunachal Pradesh. Le grand ethnologue Verrier Elwin a consacré un chapitre entier d'un de ses livres aux mythes de ces ethnies, en prenant des exemples chez les Mishmi, les Apa Tani, les Miri,

13. Dorjee, Rinjing, Tales of Uncle Tompa : The Legendary Rascal of Tibet, San Rafael, Dorjee Ling, 1975, p. 19-23. Sur la figure du trickster, la littérature est très abondante. Mentionnons Helena Bassil-Morozow, The Trickster and the System : Identity and Agency in Contemporary Society, Routledge, 2015.

14. Je remercie mon confrère Charles Ramble (EPHE, Paris) de son aide dans la collecte d'informations sur ce sujet. 
les Aka, les Gallong, etc. ${ }^{15}$. Dans cette région, les trompeurs s'affrontent entre eux, souvent à propos de la suprématie de leur groupe. Ils vont souvent deux par deux et se transforment en oiseaux ou en mammifères afin de jouer leurs tours. Ils sont à la fois très malins et stupides. Ils ne cessent de courir la prétentaine. Comme au Tibet, il s'agit avant tout de fables et de légendes. La trame narrative y est essentielle. Le religieux, en revanche, ne s'impose pas, sinon que ces personnages cumulent souvent des traits propres aux humains et aux esprits. Les tricksters appartiennent en fait à un monde très ancien où les conventions sociales étaient peu appliquées.

La duperie est également un thème central des ballades chantées/ déclamées par les bardes Dholī (une basse caste Parbatiya ou IndoNépalaise) de l'extrême ouest du Népal. Ces histoires qui sont chantées et récitées en public pour divertir l'assistance sont remplies de héros qui usent des moyens les plus malhonnêtes pour duper l'autre et parvenir à leurs fins ${ }^{16}$. Le mensonge et la ruse guident les personnages, pour la plus grande joie des spectateurs. Mais comme précédemment, l'arrière-plan religieux de ces performances orales n'apparaît pas clairement.

Les liens avec le sacré sont en revanche très puissants dans la vallée de Katmandou, au Népal. Dans cette région, la figure du trickster fait partie intégrante du patrimoine culturel des Néwar, une population népalaise de langue tibéto-birmane. Les Néwar de Patan mettent en scène tous les ans, à la même date de l'année (en novembre), trois personnages masqués et vêtus de blanc, appelés bāthah, un mot, peut-être dérivé du népali, qui signifie « rusé », « intelligent». Ils symbolisent chacun, dit-on, une des trois anciennes capitales de la vallée: Katmandou, Patan et Bhaktapur ${ }^{17}$. Les masques, décorés d'énormes moustaches, sont de

15. Elwin Verrier, Myths of the North-Eastern Frontier of India. Shillong, North-East Frontier Agency, 1958 (chapitre 8).

16. Rémi Bordes, Héros, bouffons et affligés. Anthropologie d'une poésie orale himalayenne (Dotī, extreme Ouest du Népal), Bordeaux, Thèse de doctorat, 2005, tome 1.2, p. 228-230.

17. Pour une étude approfondie de ces pièces comiques, cf. Gérard Toffin and Shova Shakya, "The Trickster in Himlayan Theatre and Folklore: Newar bathahs", Sangeet Natak Akademi Journal, vol. XLV, n ${ }^{\circ} 3-4,2011$, p. 3-32. Le livre de base (en néwari) est celui de Hariman Shrestha, Kättī pyākhã, Kārtik Nāc. Laliptur, Kathmandu, Jagadamba Press, 2009. 
couleur différente : bleu pâle pour Katmandou, couleur chair/brun clair pour Bhaktapur et brun foncé pour Patan. Ceux des bāthah de Katmandou et de Bhaktapur sont en métal, celui de Patan, le plus sacré, est fait, dit-on, de peau humaine mêlée de divers poils, dont des moustaches de tigre. Les bāthah sont présentés comme des cousins, parfois même comme les rois de leurs anciennes villes respectives. Celui de Patan, le plus jeune des trois farceurs, est réputé pour être le plus rusé et intelligent. C'est le personnage principal et son masque est crédité de pouvoirs tantriques exceptionnels ${ }^{18}$.

Le répertoire des bāthah est constitué de sept pièces comiques jouées par rotation. Chaque livret développe sa propre histoire émaillée de rebondissements rocambolesques. Des personnages secondaires, prêtres, paysannes, commerçants, eux aussi masqués, interviennent parfois. L'intrigue raconte les tours pendables que les bouffons se jouent entre eux. Les trois farceurs cherchent également à voler l'autre ou à détrousser les personnes qu'ils rencontrent sur leur chemin. Les stratagèmes sont légion. Pour parvenir à leurs fins, les bāthah inventent des histoires, mentent effrontément, tendent des pièges. Volontiers libidineux, ils séduisent les jeunes filles et brisent les ménages. Ils sont totalement amoraux et ne font qu'illustrer les travers d'hommes livrés à leurs bas instincts. Ces traits de caractère en font d'authentiques 'trickster', de joueur de tours. Le langage du corps et de l'obscène domine. Deux choses motivent les bäthah: le sexe et le ventre. Ils sont insatiables; la recherche de nourriture occupe le plus clair de leur temps. Ce sont des gloutons et leur langage est pétri d'expressions scatologiques ${ }^{19}$.

Les représentations scéniques ont lieu devant l'ancien palais royal Malla de Patan, à côté d'un temple en pierre de l'époque médiévale dédié au dieu Kṛ̣ṇa. Elles se déroulent le soir, durant trois jours consécutifs, et précèdent un théâtre religieux très

18. En 2016, ce masque était très détérioré. Mais personne à l'intérieur de la troupe n'ose le réparer ou en fabriquer un nouveau, tant il concentre en lui de pouvoirs surnaturels.

19. Les farceurs bāthah sont également connus dans la vallée de Chitlang, située sur l'une des routes pédestres qui menait autrefois vers l'Inde, ainsi qu'à Dolakha. Les potiers néwar de Katmandou mettaient autrefois eux aussi en scène des saynètes où figuraient ces bouffons. 
complexe donné en l'honneur de Varāha et de Narasiṃha, deux avatāras de Viṣnu. Ce théâtre liturgique, purānique, n'a pas de rapport direct avec les farces des premiers soirs, sinon que ses interprètes sont recrutés dans une seule et même troupe et qu'ils sont accompagnés par les mêmes musiciens. L'ensemble des ces représentations théâtrales est appelé en néwari Kāttī pyākhã (népali : Kārtik Nāc). Tous les sketches comiques sont dialogués en néwari.

Historiquement, ces comédies sont vraisemblablement nées au $\mathrm{XVII}^{\mathrm{e}}$ siècle, époque durant laquelle la vallée de Katmandou était divisée entre les trois royaumes évoqués dans les pièces. À Patan, on attribue leur création au roi Shrinivas Malla (1661-1685). Ce souverain aurait inséré des farces de ce type, bāthah pyākhã, dans les représentations Kāttī pyākhã établies par son père Siddhinarasimhha Malla. Les bouffonneries qu'il incorpora au début du spectacle proprement religieux étaient données à son époque sept jours durant, ce qui suppose un répertoire déjà considérable. Un manuscrit, depuis égaré, aurait contenu le récit de ces pièces comiques. Il est probable que ces histoires reprenaient des éléments plus anciens appartenant à la littérature orale néwar. C'est en tous les cas oralement que cette tradition s'est perpétuée au fil des siècles. Depuis les années 1970, ces livrets ont été retranscrits par écrit et imprimés dans de petits fascicules, puis dans des livres, tous en néwari.

Les aspects religieux de ces bouffonneries attirent l'attention. Les trois personnages sont souvent considérés comme des représentations des trois dieux majeurs du panthéon hindou: le farceur de Katmandou symboliserait Brahmā, celui de Bhaktapur Viṣnu et celui de Patan Śiva. Bien d'autres aspects renforcent cette dimension sacrée. Ces représentations bouffonnes par exemple ne sont pas dissociées du théâtre chorégraphique sacré, d'inspiration purānique, qui leur font suite dans le calendrier. Le lieu, la troupe, les interprètes, les instruments de musique, les chanteurs, sont les mêmes. Et c'est le directeur de l'ensemble de la troupe qui interprète généralement le bāthah de Patan. Le comique occupe les trois premières soirées, les tableaux liturgiques et sérieux (eux aussi coupés d'intermèdes comiques) sont réservés aux cinq jours suivants. Mais l'ensemble forme un tout. 
Les trois farceurs bāthah doivent par ailleurs danser en l'honneur des dieux à certains moments de la représentation. La chorégraphie, de type liturgique, ne diffère en rien des danses sacrées. Qui plus est, les bāthah s'inclinent devant Nāsahdyah, le dieu néwar de la musique et de la danse, ainsi que devant chacun d'entre eux avant de commencer leur farce. Il n'est donc pas possible de qualifier ce théâtre comique de populaire par opposition au théâtre liturgique, savant, consécutif : les deux aspects sont liés.

On peut aller plus loin dans le rapprochement. Dans leur livre récent sur les mascarades himālayennes, Pascale Dollfus et Gisèle Krauskopff cite quelques exemples (Arunachal Pradesh et Himachal Pradesh tout particulièrement) dans lesquels on attribue aux représentations théâtrales le pouvoir d'empêcher les démons de nuire aux hommes ${ }^{20}$. Un autre argument est souvent invoqué par les habitants du Toit du monde : ce type de bouffonneries aurait pour fin, principal ou secondaire, d'écarter les démons et autres mauvais esprits avant la représentation d'un spectacle religieux important. Le comique aurait donc une fonction apotropaïque. On peut d'autant plus retenir cette explication que, dans le cas qui nous occupe, les représentations de bouffons néwar de la vallée de Katmandou sont, on vient de le voir, pétries d'éléments religieux. Le déroulement du Kāttī pyākhã pourrait alors s'interpréter comme une progression spirituelle : aux premiers jours, les aspects les plus triviaux, les traits les plus bas des humains; aux jours suivants, les tableaux divins empreints de gestes nobles et de moralité supérieure. Les dieux, incarnés sur scène par des interprètes humains masqués, succéderaient au désordre et à la chienlit. Les prologues comiques n'agiraient pas comme diversion mais plutôt comme conjuration des mauvais esprits.

Les bāthah néwar évoquent le bouffon/clown vidūșaka de l'Inde classique sanskrite. Les deux figures comiques partagent de nombreux traits communs: le vidūsaka, comme les bäthah, fait rire, il peut être méchant et faire des tours peu recommandables, il séduit les femmes, son rapport avec la royauté est étroit, il est bavard, rusé, il a la face tordue (comme un des trois clowns néwar). Le parallèle est particulièrement frappant car ces clowns de l'Inde ancienne - toujours présentés comme des brāhmanes - jouent un 
rôle important, quoique discuté, dans le théâtre sanskrit classique. Le philologue F.B.J. Kuiper y voyait une personnification déguisée de Varuṇa, en opposition conflictuelle avec Indra ${ }^{21}$. D'après lui, le vidūssaka, auquel le vieux traité du théâtre Nātyaśāstra accorde un rôle important, assume dans le drame sanskrit un rôle religieux, cosmogonique, qui dépasse sa fonction strictement comique ou burlesque. Il représenterait les forces contraires au bon déroulement de la performance théâtrale, les obstacles à la marche des cérémonies religieuses. Le vidūṣaka en définitive incarnerait un 'contre-théâtre'. C'est la raison pour laquelle il est présenté dans le Nātyaśāstra comme un adversaire du nāyaka, le maître d'œuvre de la pièce ${ }^{22}$. Le bouffon déchargerait aussi le roi, le sacrifiant et le commanditaire principal du théâtre hindou classique de cour, des péchés et de l'impureté liés au métier de souverain. Il serait une sorte de bouc émissaire ${ }^{23}$.

Il n'est pas anodin que le vidūṣaka intervienne dans le Nātyaśāstra au début de la représentation théâtrale, lors des rituels préliminaires pūrvarainga, de la même manière que les bāthah néwar se produisent avant le début de la pièce religieuse proprement dite. Il est également frappant que les clowns néwar soient incorporés à la pièce, qu'ils apparaissent comme un élément indissoluble de la représentation Kāttī pyākhã, comme le bouffon indien, et non comme un élément extérieur, juste là pour faire rire. Les clowns néwar bāthah donnent du sens et du crédit à l'interprétation religieuse de Kuiper.

Les aspects royaux liés à ces trois farceurs sont eux aussi marqués. Traditionnellement, c'était le roi Malla de Patan qui incarnait le troisième băthah, le plus jeune des bouffons. Longtemps ce fut du reste à un descendant de la famille royale que revenait cette fonction. Aujourd'hui encore, la troupe reste sous la tutelle d'une ancienne famille Śreștha hindoue, associée à la fonction royale, et de leurs prêtres Rajopadhyaya néwar. Le masque mystérieux du bāthah de Patan connote les sacrifices

21. F.B.J. Kuiper. Varuna and Vidūshaka. On the Origin of the Sanskrit Drama. Amsterdam, North-Holland Publishing Company, 1977. Sur le vidūṣaka, voir aussi Lyne Bansat-Boudon, Poétique du théâtre indien. Lectures $d u$ Nātyashāstra, Paris, École Française d'Extrême-Orient, 1992, p. 268.

22. Idem, p. 210.

23. Idem, p. 233. 
humains et la sauvagerie, des traits royaux paradigmatiques dans les conceptions hindoues. David Shulman a admirablement montré, dans son livre The King and the Clown, les liens entre royauté et clownerie dans le sud de l'Inde médiéval. Le rapprochement avec la vallée de Katmandou est, ici aussi, saisissant. Qu'en Inde les brāhmanes jouent le rôle de bouffon renforce le rapprochement entre les deux régions. Comme les rois hindous du pays tamoul ${ }^{24}$, les trois bāthah sont censés par ailleurs être tombés du ciel et observer avec attention ce qui se passe sur terre. D'une manière générale, rappelons-le, dans la culture du carnaval, le bouffon est presque toujours une figure du roi, détrôné pour l'occasion ${ }^{25}$.

C'est à la lumière de ces vieilles représentations indiennes, identifiant les rois à des bouffons, que les bäthah néwar prennent tout leur sens. Elles décrivent un roi sans réel pouvoir, jouet des passions sexuelles et des volontés divines. De même, dans les ballades et épopées chantées encore aujourd'hui dans l'extrême ouest du Népal, les rois sont allègrement moqués. Ce sont des fanfarons, des personnages excessifs aux formules ampoulées, des gloutons qui ne pensent qu'aux mets épicés qu'on va leur servir. Ils ne parviennent à leur fin qu'en se déguisant pour tromper les gens $^{26}$. Comment ne pas penser ici à Indra ? Ce dieu, prototype de la royauté, n'est-il pas exhibé lors de sa fête de Katmandou sous des traits comiques, à la limite de la bouffonnerie ? ${ }^{27}$ Dans cette fête et dans la légende qui lui est associée, Indra, le roi des dieux, est un anti-héros qui se cache dans une fleur de lotus pour éviter d'être capturé tel un voleur dans un jardin de Katmandou ${ }^{28}$. Il est finalement jeté en prison par les autorités. Ce sont là des aspects peu glorieux, inattendus, qui donnent de la royauté hindoue une image plus complexe que celle que l'on se fait d'habitude.

24. David Shulman, The King and the Clown in South Indian Myth and Poetry. Princeton, Princeton University Press, 1985, p. 295.

25. Mikhaïl Bakhtine, L'Euvre de François Rabelais et la culture populaire au Moyen Âge. Paris, 1970, Gallimard, p. 199.

26. Rémi Bordes, Héros, bouffons et affligés, op. cit., p. 229.

27. Gérard Toffin, La fête-spectacle. Théâtre et rite au Népal. Paris, Éditions de la Maison des Sciences de l'Homme, 2010, Chapitre 3.

28. Ce thème de la dissimulation, de la cachette est également très présent dans les chants bhärat de l'extrême ouest du Népal ; cf. Bordes, op. cit., p. 230. 


\section{LES CLOWNS BRĀHMANES ET ASCÈTES HINDOUS}

S'il est bien un trait commun aux mascarades et autres bouffonneries collectives de l'Himālaya, c'est le rôle comique qu'elles octroient aux prêtres brāhmanes et aux ascètes hindous désignés sous le nom de sannyāsī ou de jogi (yogī, bābājī). Le thème est courant au Népal, dans l'Himālayen indien occidental (Kumaon et Garhwal) et oriental (Arunachal Pradesh), au nord comme au sud. Rémi Bordes a collecté dans l'extrême ouest du Népal quantité de chants et de ballades qui mettent en évidence ce trait dans la culture populaire Parbatiya à tradition orale ${ }^{29}$. J'ai eu, pour ma part, maintes fois l'occasion d'observer dans des villages néwar des pièces comiques dans lesquelles un brāhmane éméché et titubant sous l'effet de l'alcool, pourtant interdit à sa caste, ne parvenant plus à se remémorer les formules mantra et les rituels nécessaires à la célébration d'un mariage. Son embarras et son éthylisme provoquent l'hilarité des spectateurs. Les brāhmanes, représentant des valeurs religieuses les plus hautes, sont donc moqués. De semblables satires existent chez les Gurung et les Tamang, deux groupes ethniques népalais de langue tibéto-birmane ${ }^{30}$.

Les jogi, sannyāsī et autres renonçants hindous ne sont pas mieux lotis. Eux aussi sont peints lors des mascarades et des représentations théâtrales sous forme de personnages comiques, aux identités variées, souvent grivois et obsédés par le sexe. De fait, ce personnage de l'ascète hindou, les cheveux tirés au sommet de la tête en un chignon, son bol à aumônes à la main, voué à une perpétuelle errance, vivant en solitaire, lui qui a quitté sa famille mais qui a adopté en retour l'humanité tout entière, et qui passe son temps à fumer des substances intoxicantes, aux frontières de la folie, semble avoir marqué les imaginations ${ }^{31}$.

29. Rémi Bordes, Héros, bouffons et affligés. op. cit., tome 1.2

30. On se moque aussi des brāhmanes, par exemple de leur supposée abstinence, souvent transgressée, en Inde. Olivier Herrenschmidt rapporte à ce sujet un certain nombre d'historiettes que les villageois de l'Andhra Pradesh se racontent, en pouffant de rire. Cf. Les dieux hindous sont les meilleurs, 1989, p. 32-34. Les brāhmanes y apparaissent comme obscènes et lubriques.

31. Dans l'extrême ouest du Népal, les ascètes jogi sont souvent peints dans les chants bhärat sous les traits les plus noirs : dissimulation, escroquerie, magie noire, etc. Cf. Rémi Bordes, 2005, t. 1.2, p. 207. Le jogi n'est qu'un dissimulateur, un travesti opportuniste. 
Chez les Néwar des collines népalaises, les danseurs incarnant les figures démoniaques lākhay (voir infra) sont souvent accompagnés d'acolytes déguisés en jogi ou de clowns masqués portant des éléments associés à la vie des ascètes, tels des plants de Datura, une plante psychotrope de la même famille que la mandragore et la belladone, connue pour ses effets toxiques et fréquemment utilisée par les yogis, en particulier les ascètes Aghori vivant près des lieux de crémation ${ }^{32}$. Toujours chez les Néwar, les joueurs de tours bāthah possèdent eux aussi des éléments ascétiques dans leur accoutrement, tel le chignon noué sur le sommet du crâne. Des ascètes interviennent aussi dans la suite du spectacle religieux du Kärtik Nāc, mais ceux-là font partie des allégories religieuses dites sérieuses. Signalons enfin que lors des carnavals du Sā Pāru (fête de la vache) et du Matayāh, en juillet-août, au cœur de la saison des pluies, des enfants déguisés en jogi, vêtu d'une robe safran, couleur emblématique de l'ascétisme, un collier de graines de rudrākșa au cou, caractéristiques des ascètes śivaïtes, des bols à aumônes à la main, participent aux parades funéraires organisées par les familles qui ont perdu un parent dans l'année ${ }^{33}$. Mais, comme dans le cas précédent, le déguisement est moins destiné à faire rire qu'à porter l'allégresse générale à son comble.

À examiner de près la littérature ethnographique, on s'aperçoit que le terme jogi s'applique à un très grand nombre de personnages comiques, avec ou sans traits ascétiques. Il est synonyme de clowns. Ainsi chez les Gurung, Pirkko Moisala décrit des performances mi religieuses mi profanes appelées sorathi $\bar{l}$, durant lesquelles se jouent des intermèdes comiques, appelés localement jogi laba, particulièrement intéressants ${ }^{34}$. Un homme déguisé en jogi porte un masque en bois et joue plusieurs personnages: astrologue, forgeron, pêcheur. J'ai observé des scènes identiques dans la haute vallée de l'Ankhu Khola, chez les

32. Gérard Toffin, Living Masks of the Newars. The Itinerant Masked Dances of the Kathmandu Valley, Paris, La lettre du Toit du monde, 13, Paris, 2014, p. 20.

33. Gérard Toffin, "Chemins du rire et des morts; carnaval, burlesque et satire au Népal», in Nathalie Gauthard (éd.), Fêtes, mascarades et carnavals. Montpellier, Éditions L'Entretemps, 2014, p. 110.

34. Pirrko Moisala, Cutural Cognition in Music. Continuity and Change in the Gurung Music of Nepal, 1991, Turku University, Suomen ethnomikologisen seuran julkaisuja, 4, Jyväskyalä., p. 341 et figure 21. 
Tamang du Ganesh Himal (Népal), un groupe ethnique qui parle une langue apparentée aux Gurung (et aux Thakali). Lors des fêtes collectives mane shyaba, des jogi, le visage passé à la suie, le corps emplumé surgissent on ne sait d'où et courent après les jeunes filles en faisant des gestes obscènes. L'un d'eux asperge de boue l'assistance ${ }^{35}$.

Dans le district de Kullu, en Himachal Pradesh (Inde), des mascarades, accompagnées par des musiciens de basse caste, se déroulent chaque année en hiver au début du mois de Phag (févriermars). Ces festivités ont lieu durant la fête de Phalgi dédiée à des divinités viṣnuïtes ${ }^{36}$. Les personnages masqués qui dansent et paradent lors de ces manifestations portent le nom de rși et rșa. Ce sont des figurations d'ermites hindous, à la fois masculins (pour cinq d'entre eux) et féminin (un seul). Leur statut est plus ou moins similaire à celui des renonçants. Cette mascarade évoque les cinq Pāṇ̣̂ava et Draupadī, héros du Mahābhārata, un thème mythologique omniprésent dans cette partie de l'Himālaya indien. Les masques, aux yeux fortement en amande et portant un losange religieux au milieu du front, sont en bois peint. On a affaire ici à une illustration d'un thème purānique. Les divinités et les figures religieuses ne sont pas directement moquées. Ces performances sont assorties de simulacre de labour et de copulation entre les personnages masqués, probablement des rites de fécondité et de prospérité, souvent associés aux fêtes marquant les passages de saison.

Chez les Thakali de la vallée de la Kali Gandaki, au Népal, des jeunes garçons déguisés en jogi interviennent lors des saynètes qui se tiennent à l'occasion de la fête annuelle de Phala (août-septembre). Ils ont le corps frotté de cendres et le visage marqué des signes caractéristiques des ascètes hindous ${ }^{37}$. Avec des morceaux de bois taillés en forme de pénis, ils s'expliquent les uns les autres comment les faire pénétrer dans un vagin, dans différentes positions. Prise à partie pour toucher et essayer à son

35. Gérard Toffin, «Au Népal, les Tamang, ces hommes qui dansent leur histoire », Notre Histoire, 31, 1987, p. 54.

36. Cf. Michael Vinding, The Thakali, A Himalayan Ethnography, London, Serindia Publications, 1998, p. 338. Voir aussi Pascale Dollfus \& Gisèle Krauskopff, Mascarades, op. cit., p. 130-137.

37. Dollfus et Krauskopff, op. cit., p. 140. 
tour le pénis en bois, l'assistance s'esclaffe de rire. Ces sketches sont appelés localement jogi lyāngpā, littéralement «jouer au jogi $\gg$. Ces clowns bénissent aussi les maisonnées du village en tournant trois fois autour du pilier central de l'habitation. En retour, ils reçoivent de l'alcool et de la nourriture ${ }^{38}$.

Des jogi interviennent également dans les représentations théâtrales jouées par les Rajbanshi dans l'extrémité orientale du Tarai népalais et plus à l'est encore, dans les basses terres d'Assam. Ce théâtre tire son inspiration du Rāmāyaṇa, l'épopée indienne, et met en scène ses principaux héros. Les jogi dans ces tableaux apparaissent sous la forme de bouffons, le visage enfariné comme des clowns, vêtus d'orange avec une perruque de même couleur figurant le chignon noué des ascètes, un sac en bandoulière et une pincette à feu à la main ${ }^{39}$. Ils font rire l'assistance, contrairement aux grandes figures du Rāmāyaña qui, elles, admettent peu la parodie. Ces jogi ne sont pas masqués mais simplement grimés.

Ce rôle comique dévolu aux brāhmanes et aux ascètes n'est pas spécifique à l'Himālaya. Il apparaît dans maints théâtres comiques populaires de l'Inde. Comment expliquer que les représentants des valeurs ultimes soient ainsi tournés en dérision et pris en si mauvaise part le temps d'une fête ou d'une représentation théâtrale ? Le bouffon vidūṣaka du théâtre indien classique lui-même, on l'a vu, était un brāhmane. Bossu, difforme, peureux, il ne pense qu'à manger et raye continûment le roi et ses folies amoureuses. Tout se passe comme si les membres de la plus haute caste de la société hindoue étaient prédisposés, du fait même de leur haute position, à jouer les rôles burlesques sur la scène. Il en était ainsi dans l'Europe médiévale durant les fêtes des fous, où les prêtres menaient la danse. Il en est de même dans bien d'autres régions du globe. Le temps du carnaval, le haut devient le bas. Quant à l'ascète sannyāsī qui se met volontairement au ban de la société normée, il se trouve lui aussi en position d'être assimilé en certaines occasions aux castes les plus basses et d'être peints sous des traits obscènes.

Cette proximité du bouffon avec l'état de brāhmane a persisté à travers les âges. Il survit aujourd'hui dans le théâtre kūtiyāttam du Kerala, où des clowns, la tête drapée dans un large turban rouge,

38. Vinding, op. cit., p. 338.

39. Dollfus et Krauskopff, op. cit., p. 203. 
de larges boucles d'oreille rondes aux oreilles, une moustache aux extrémités effilées, le visage peinturluré de marques blanches, portent le dhoti et le cordon sacré des deux-fois-nés. Ce personnage s'exprime en langue populaire prakrit, il décrit la corruption de la société et la folie des hommes. Son rôle est satirique $^{40}$. Les farces succèdent aux pitreries et nécessitent parfois l'utilisation de phallus postiches. C'est là un héritage de la culture sanskrite ancienne, bien oublié de nos jours. On aurait du mal à trouver des références à de telles figures comiques dans le livre de Louis Dumont Homo hierarchicus, pourtant centré sur le brāhmane et le roi en Inde. Ce topos ludique démystifie pourtant ces figures canoniques hindoues.

\section{DIVINS FRIPONS ET BOUFFONS SACRÉS TIBÉTAINS, ATSARA}

Dans plusieurs régions de culture et de langue tibétaine, les représentations de théâtre ache lhamo et les danses monastiques masquées cham sont entrecoupées d'épisodes comiques joués et dansés par des atsara, un mot dérivé du sanskrit ācārya et qui signifie : «maitre religieux », «savant», «brāhmane $»^{41}$. Ces atsara sont des clowns (on peut dire aussi des farceurs), vêtus de costumes bariolés, proche des vêtements en loque des mendiants. Ils ont le visage caché par des masques au nez très proéminent et ils s'emploient à divertir le public par diverses pitreries. Ces clowns interviennent généralement par groupe de deux ou de quatre. Dans le cham la structure narrative des pièces comiques est plus réduite que dans la tradition ache lhamo. Mais dans un cas comme dans l'autre, la représentation fait une large part à l'improvisation. De telles scènes comiques ouvrent parfois, tel un prologue, le

40. David Shulman, The King and the Clown, op. cit., pp. 175-176. Des faits similaires existent en pays tamoul, idem, p. 194.

41. Dans son livre posthume sur les danses cham, Tibetan Religious Dances. Text and Translation of the 'Chams yig. La Haye, Mouton 1976, René de NebeskyWojkovitz mentionne à plusieurs reprises des danses clownesques atsara sans toutefois dégager leur significations religieuse. Parfois, il emploie seulement le mot 'joker' pour désigner ces personnages masqués. Certaines saynètes (cf. par exemple p. 17) semblent de fait purement séculières et n'ont pas d'autre but que de distraire l'auditoire. Ces figures comiques ne figurent pas dans les textes en tibétain qui contiennent des instructions sur la chorégraphie des danses cham. 
spectacle. Dans les danses monastiques de type cham, ce sont les moines eux-mêmes qui interprètent les rôles de clowns ${ }^{42}$.

À la fin du XIX ${ }^{\mathrm{e}}$ siècle, Austin Waddell précise que les «maittres » bouffons, $\bar{a} c \bar{a} r y a s$, tibétains portent des petits masques en tissu « de taille ordinaire » et de couleur blanche, brune ou noire lors des danses et représentations de théâtre ${ }^{43}$. Leur chevelure est peinte en bleu et ramenée dans un chignon porté sur le sommet de la tête comme les yogīs indiens. Ils sont les bouffons et les clowns de la pièce et se produisent souvent durant les intermèdes. Ailleurs, Waddell précise que dans les représentations théâtrales achelha-mo, des bouffons incarnant des brāhmanes et des « chasseurs » (aux masques bleus sertis de cowries et jupettes en cordes de poils de yak) interviennent lors des interludes ${ }^{44}$.

Dans une étude beaucoup plus récente centrée sur le monastère nyingmapa de Shechen, à Bodnath, Népal, Nathalie Gauthard nous apprend que les masques de ces clowns sont faits de plusieurs couches de tissu collées ou en bois qui recouvrent tout le visage. L'expression esquisse un sourire, dévoilant deux rangées de dents blanches. Des ballons en plastique gonflables sont fixés au masque. Ils se moquent de tous, et d'abord d'eux-mêmes. Ils singent aussi le pas des moines en mimant un coït anal. Dans ce monastère de Shechen, les atsara peuvent aussi être amenés à assurer le service d'ordre durant les représentations de théâtre dansé. Ils canalisent la foule qui a tendance à déborder sur l'aire de danses des moines lors des représentations cham. Ils servent aussi d'accessoiristes et de costumiers. Ils sont parfois chargés de faire la promotion de gestes écologiques, comme le ramassage des bouteilles en plastique ${ }^{45}$.

42. Dans certains cas, les danseurs de chorégraphies cham peuvent être des laïcs. Cf. Pommaret, "La danse des 'huit catégories de dieux et démons' au Bhoutan », in K. Buffetrille \& H. Diemberger (eds), Territory and Identiy in Tibet and the Himalayas, Leiden, Brill, 2002. p. 170-171. Par ailleurs, lorsque la cour du monastère est trop petite, les clowns atsara peuvent se produire ans l'espace villageois.

43. Austin Waddell, Buddhism and Lamaism of Tibet. Delhi, 1974 (1895), Heritage Publishers, p. 538.

44. Idem, p. 540

45. Nathalie Gauthard, «Atsaras, incarnation de la «folle sagesse» du bouddhisme tibétain. Entre rire et transgression», La croyance et le corps, Esthétique, corporéité des croyances et identités, Bordeaux, Presses Universitaires de Bordeaux, 2015, p. 235-243. 
En fait, ces clowns ne se réduisent pas (ou pas toujours) à leurs fonctions comiques. Ce sont des personnages complexes qui concentrent en eux plusieurs idées et conceptions. Ils peuvent avoir à l'occasion des fonctions religieuses, celles notamment d'écarter et de mettre à mort les démons. Selon Rolf Stein, les clowneries, plaisanteries qui agrémentent les fêtes tibétaines (comme celles du nouvel an) ont aussi pour but d'amuser les dieux et de produire un effet bénéfique sur les récoltes et le bétail ${ }^{46}$. Dans certains villages du Bhoutan, les atsara peuvent être conçus comme l'incarnation des divinités protectrices de la vallée. Certains atsara sont assimilés au saint bhoutanais Drukpa Kunley ou à un personnage de la vie mythique de Yeshe Tsogyal, la parèdre du grand saint Padmasambhava ${ }^{47}$.

Toujours au Bhoutan, les atsara ont des phallus en bois et des ballons en vessie de porc. Dans ce pays les clowns prennent des formes carrément priapiques et scatologiques que les jeunes femmes redoutent. De plus ils quémandent de l'argent au public. Dans ce pays, Françoise Pommaret a décrit des danses cham dans lesquelles des atsara aux costumes et masques rouges brandissent un phallus en bois ${ }^{48}$. Ils interviennent directement dans les parties sacrées de la performance pour tuer symboliquement les démons et ils imitent de façon comique l'oracle-médium $d p a$ 'bo. La glose locale les voit volontiers comme des serviteurs des divinités incarnées dans la danse. Mieux encore : les phallus qu'ils tiennent à la main leur servent à bénir les femmes qui veulent avoir des enfants ${ }^{49}$. De son coté, Michael Arris a décrit une fête bhoutanaise dans laquelle un clown touche les femmes qui assistent au spectacle avec un phallus postiche ${ }^{50}$.

46. Cf. Rolf Stein, Recherches sur l'épopée et le barde au Tibet, Paris, PUF («Bibliothèque de l'Institut des hautes études chinoises », vol. XIII), 1959, p. 443.

47. Cf. F. Pommaret et Tashi Tobgay, "Bhutan's Pervasive Phallus : is Drukpa Kunley really Responsible ?", in A. McKay \& A. Balikci-Denjongpa (eds.), Buddhist Himalaya: Studies in religion, History and Culture, Gangtok, Namgyal Institute of Tibetology, 2011, vol. 1, p. 059-76.

48. Pommaret, "La danse des 'huit catégories de dieux et démons' au Bhoutan », op. cit., p. 170.

49. Cf. Pommret et Tashi Tobgay, op. cit., p. 65.

50. Micheal Arris, "The admonition of the thunderblot cannonball and its place in the Bhutanese New year Festival" Bulletin of the School of Oriental and African Studies, vol. XXXIX, 3, 1976, p. 606. 
Ces clowns se livrent très fréquemment à une parodie de la religion. Les railleries sont nombreuses et s'attaquent aux éléments déviants des religieux, aux manquements à la vertu supposée: satire des oracles ineptes qui ne savent rien dire ni prévoir par exemple ou des mœurs sexuelles déréglées des moines et des nonnes qui ne pensent qu'à soutirer de l'argent de leurs commanditaires. Le blasphème pourtant n'existe pas et les critiques épargnent les grands maîtres et le Dalaï-Lama ${ }^{51}$.

Certaines exégèses accordent aux atsara une signification religieuse encore plus élevée. Les atsara seraient proches des saints fous (nyönpa) des yogīs bouddhistes apparus au $\mathrm{XIV}^{\mathrm{e}}$ siècle, qui s'habillaient de manière excentrique et rejetaient les comportements traditionnels du moine bouddhique. Selon les autorités religieuses tibétaines, ils descendraient des mahāsiddha, «les grands accomplis» indiens, personnages religieux qui ont achevé de hauts degrés d'élévation et d'initiation mystique et qui seraient apparus entre le $\mathrm{VII}^{\mathrm{e}}$ et le $\mathrm{XII}^{\mathrm{e}}$ durant l'époque $\mathrm{du}$ développement du bouddhisme tantrique Vajrayāna en Inde et au Tibet. Ivres de religion, ils manifesteraient leur état par la danse et le chant ${ }^{52}$. Ces saints fous représenteraient une sorte de «folle sagesse» (ye shes 'chol ba). Les nyönpa mettraient la foi à l'épreuve en ouvrant la voie à des niveaux supérieurs de connaissance, libérés de tout attachement et de tout comportement mondain. Ces religieux non conventionnels venaient essentiellement des couches populaires. Certains avaient la réputation d'être de grands buveurs et de fréquenter les maisons de plaisirs $^{53}$.

Les atsara sont également les incarnations de l'étranger, du non-tibétain, presque toujours indien ${ }^{54}$. Au XVIII ${ }^{\mathrm{e}}$ siècle, le mot désignait tous les visiteurs indiens au Tibet ${ }^{55}$. En Inde même, surtout dans les sources coloniales, le mot ācārya céda la place

51. Idem, p. 486.

52. Rolf A. Stein, Vie et chants de 'Brug-pa Kun-legs, le yogin, traduit et annoté du tibétain, Paris, Maisonneuve et Larose, 1972, p. 8

53. Nathalie Gauthard, idem, p. 236.

54. Rolf A. Stein, «Le thêâtre au Tibet », in Jean Jacquot (éd.), Les Théâtres d'Asie, CNRS, Paris, 1978, p. 136.

55. Toni Huber, The Holy Land Reborn. Pilgrimage and the Tibetan Reivention of Buddhist India, Chicago, Chicago University Press, 2008, ch. 7, p. 196. 
au terme Gosain, qui s'appliquait à tous les mendiants religieux ainsi qu'aux marchands pèlerins religieux. Les atsara sont donc un pendant des clowns yogis qui se produisent en Inde. À l'identité religieuse s'ajoute un symbole d'altérité. Ils incarneraient l'opposition traditionnelle entre peuples de plaines et ceux des montages $^{56}$.

Isabelle Henrion-Dourcy, une spécialiste du théâtre ache lhamo tibétain, corrobore le lien entre atsara et altérité ${ }^{57}$. Elle l'étend du reste à d'autres types de populations que les Indiens. Les bouffons de ce théâtre si populaire autrefois au Tibet figurent ainsi des musulmans avec une petite toque sur la tête, des savates aux bouts relevés aux pieds (tels les bateliers dans la pièce Sukyi Nyima), ou des chinois (autres bateliers dans Pema Obar) avec un petit chapeau à rabats, un pantalon de soie noire ${ }^{58}$. Ces étrangers sont dépeints avec force moquerie et stéréotypes (salamalecs, commerce à tous crins). Leurs déhanchements sur scène déclenchent de grands éclats de rire dans l'assistance ${ }^{59}$. Quant aux danses cham, interprétées par les moines dans l'enceinte des monastères, le bouffon incarne souvent le personnage de l'anachorète chinois Hashang. Dans le cas du ache lhamo, ces pitreries en général ne sont pas inclues dans le livret mais elles brodent sur des canevas bien connus ${ }^{60}$.

\section{LES BOUFFONS CHAMANIQUES}

Le chamanisme, qui, à la différence des simples cultes de possession, nécessite un long processus d'initiation, l'apprentissage

56. Là-dessus ainsi que sur l'atsara en général, voir: Nathalie Gauthard, Atsara, le maître-moqueur. Les métamorphoses du rire tibétain contemporain, HDR, Paris-Sorbonne, 2014, tome 2,

57. Isabelle Henrion-Dourcy, "Le théâtre tibétain ache lhamo : un contenu d'héritage indien dans des formes d'héritage chinois ", in Hélène Bouvier et Gérard Toffin, Théâtres d'Asie à l'œuvre. Circulation, expression, politique, Paris, Éditions de l'EFEO, 2014, p. 90.

58. Isabelle Henrion-Dourcy, Ache Lhamo. Jeux et enjeux d'une tradition théâtrale tibétaine, 2 vol. Université libre de Bruxelles/École pratique des hautes études, 2004, vol. 1, p. 379.

59. Isabelle Henrion-Dourcy, «Le théâtre tibétain ache lhamo... », 2014, op. cit., p. 90.

60. Isabelle Henrion-Dourcy, Ache Lhamo, 2004, op.cit., p. 484. 
de chants et de psalmodies, et qui suppose un long voyage semé d'embûches du chamane dans l'au-delà, est un phénomène central de la vie religieuse d'une grande partie des populations de l'Himālaya central (Népal en particulier) et oriental. Les chamanes officient la plupart du temps seuls. Parfois ils sont accompagnés d'assistants, apprentis chamanes ou chamanes confirmés. Il peut arriver aussi qu'ils se produisent en groupes, par exemple lors des pèlerinages et des fêtes collectives. On les sollicite pour chasser les maladies, dénouer les affaires de sorcellerie, partir à la recherche d'une âme.

Les témoignages sur la participation de bouffons aux séances chamaniques sont rares. Dans la très grande majorité des cas, ce type de personnage comique, spécialisé, n'est pas requis. Les chamanes avec lesquels j'ai travaillé, en milieu tamang (Népal) par exemple, ont coutume de dire que le rire est malvenu dans les voyages chamaniques. Ces séances exigent du sérieux, beaucoup d'attention, la récitation de longues psalmodies, elles impliquent des combats avec les mauvais esprits qui menacent les intercesseurs de maléfices. Tout ceci exclut les pitreries et autres bouffonneries. Un film récent (2015) de deux ethnographes amateurs et cinéastes français, Aurore Clément et Adrien Viel, Trois chamanes, rencontres chamaniques au Népal, (Hong Kong Connection et Epicerie films, 2014) confirme, de la bouche même d'un des chamanes filmés (Ghale), ce point de vue.

Il existe cependant des exceptions qu'il faut considérer avec attention car elles révèlent des conceptions symboliques qui s'étendent à mon avis bien au-delà du chamanisme. Le cas le mieux documenté est celui des chamanes Kham Magar du Népal étudiés par Michael Oppitz et Anne de Sales. Cette dernière, une ethnologue française, a décrit en détail les pitreries qu'accomplit pendant les séances chamaniques un personnage appelé localement lāta, du mot népali lāto qui désigne le «simple d'esprit», le «muet», en népali. En tête de son étude, Anne de Sales esquisse une comparaison entre le bouffon et le chamane: tous deux, dit-elle, sont des passeurs de frontières et des transgresseurs de catégories ${ }^{61}$. L'auteur passe ensuite aux détails ethnographiques.

61. Anne de Sales, «Chamanes et bouffons (Népal et Sibérie)», Études mongoles et sibériennes, 26, 1995, p. 18. 
Ce läta a pour attribut rituel principal un phallus de bois, à l'extrémité duquel est accrochée une touffe de cheveux qui évoque les poils pubiens du sexe féminin. Ce phallus peut gagner en réalisme avec l'ajout d'une paire de testicules de bois. Le corps de l'idiot est peinturluré de cercles, de rayures et de zigzags blancs, avec une solution de craie et d'eau. Il porte des vêtements de couleur bigarrée, il a une couronne de feuilles sur la tête, une cloche est attachée à sa ceinture.

C'est dans cet accoutrement, déjà très élaboré, que le bouffon intervient lors des cérémonies collectives de consécration d'un nouveau chamane. Il gesticule en tête de la procession des intercesseurs jhānkri, poussant des cris, montrant son derrière, mimant l'acte sexuel et menaçant les femmes de son phallus en bois. Les Kham Magar disent que le lāta effraye les mauvais esprits, terrifiés à la vue d'un organe sexuel si évocateur, eux qui ne procréent pas. Ces clowns rituels éloigneraient donc les démons, comme les sifflements, dit-on dans l'aire culturelle tibétaine, auraient des pouvoirs démonifuges ${ }^{62}$. Le bouffon ouvrirait en quelque sorte la voie aux chamanes, il serait là pour faire fuir les démons ou pour les distraire, le temps que le chamane récupère les âmes humaines qu'ils ont volées ${ }^{63}$. La couleur blanche dont il s'enduit le corps aurait le même effet sur eux que le phallus. Cette couleur éblouit les êtres surnaturels malfaisants ainsi que les maîtres du monde sauvage et de l'obscurité.

Les mêmes thèmes et les mêmes accessoires se retrouvent lors des séances de guérison pour lesquelles le chamane est appelé dans les maisons. Le lāta est encore là, à côté du chamane et il participe activement au déroulement des rites. Parfois ils sont deux, l'idiot et l'idiote, lāta, lāti, le rôle de la femme étant joué par un homme travesti portant une longue jupe. Dans ce cas, le phallus est exclu. Sinon, le bouffon utilise son phallus de bois pour soulever les coins de la natte où repose le patient, geste qu'Anne de Sales

62. Françoise Pommaret, «Maîtres des trésors» (gter-bdag): divinités locales et médiums au Bhoutan », A.M. Blondeau (éd.), Tibetan Mountain Deities. Their Cults and Representations, Verlag der Osterreichischen Akademie der Wissenschaften, 1988, p. 90.

63. Cf. Anne de Sales, "The go-between : Reflections on a mechanism of ritual exchange", in C. Gerschlager (ed), Expanding the Economic Concept of Exchange. Deception, Self-deception and Illusions, Boston, Kluwer Academic Publishers, 2001, p. 126. 
interprète comme un moyen d'ouvrir la voie à l'âme du malade et de lui frayer un chemin vers le monde des humains. Là aussi, ce personnage comique aurait pour fonction de repousser les mauvais esprits qui pourraient obstruer les voies entre ciel et terre et de rétablir une communication contrariée entre les différents étages du monde ${ }^{64}$.

Ce bouffon est interprété tantôt par des gendres de la famille, tantôt par un forgeron Kāmi de basse caste Parbatiya (IndoNépalais) ${ }^{65}$, une caste très présente dans ces régions reculées du massif du Dhaulagiri où vivent les Kham Magar - les Kāmi sont aussi fort nombreux parmi les Tamang du Ganesh Himal, plus à l'est. C'est là un fait fondamental que l'on retrouve ailleurs en Himālaya, dans les plaines du Terai comme dans les zones montagneuses: le bouffon est souvent choisi parmi des personnes de bas statut, car chez elles la liberté d'expression et de mouvement, les grimaces, les rires sans retenue, la franchise sans détours de leur propos, la propension au comique en résumé apparaît congénitale aux yeux des hautes castes. Cette asymétrie sociale ne doit jamais être oubliée dans l'interprétation des rituels chamaniques.

Selon Anne de Sales, le bouffon aurait un rôle central dans ces cérémonies. Cet assistant consubstantiel du chamane serait à la fois «libérateur et régénérateur ${ }^{66}$. De Sales qualifie de «renversements carnavalesques $»^{67}$ les postures auxquelles ce clown se prête, des postures qui feraient partie intégrante de la séance. Michael Oppitz a publié des photos de ce bouffon Kham Magar (appelé dans le village où il a travaillé lātā kukur, « le chien muet») et de son phallus qui corroborent la description d'Anne de Sales et qui ajoutent des éléments visuels particulièrement saisissants ${ }^{68}$. Oppitz suggère que le rire aurait pour fonction

64. Ces clowns lāto font aussi penser aux idiots de village qu'a étudiés Margarita Xantakou selon une perspective psychologique dans le Magne, dans le sud du Péloponnèse. Cf. Margarita Xantakou, Idiots de village, conversations ethnopsychiatriques en Péloponnèse, Toulouse, Presses Universitaires du Mirail, 1989.

65. P. Dollfus \& G. Krauskopff, op. cit., p. 92.

66. Anne de Sales, « Chamanes et bouffons », op cit., p. 25.

67. Idem, p. 25.

68. Michael Oppitz, "Drawnings on Shamanic drums: Nepal", Res: Anthroplogy and Aesthetics, 22, 1992, p. 78, fig. 28. Sur cette photo, on voit une cloche accrochée à l'extrémité du pénis. 
d'attirer les mauvais esprits, de les débusquer en quelque sorte ${ }^{69}$. Il serait alors plus facile à capturer. Ce n'est cependant pas le chamane qui joue ce rôle. La dissociation des fonctions suppose une disjonction entre rire et sacré, liturgie et comique. Par ailleurs ce sont souvent des personnes extérieures au groupe Kham Magar, des hommes de basses castes, qui interprètent ces personnages comiques. Il est donc difficile de leur assigner un rôle central dans un système religieux qui leur est partiellement étranger et dont la langue religieuse est basée autant sur le kham magar que sur le népali.

L'association entre comique et chamanisme existe ailleurs. Des peuples turcs de l'Altaï tels les Koumandines, les Téléoutes et les Chors, font intervenir des bouffons, appelés Paktu Khan ou Koca Khan (ou Kan), lors des cérémonies chamaniques ${ }^{70}$. Les chamanes ont un phallus comme attribut, avec des testicules en bois attachées à son extrémité. Ils s'en servent pour repousser les mauvais esprits ${ }^{71}$. Eux aussi ont des costumes bariolés, rayés ou tachetés. Lors de fêtes agraires saisonnières, le koca khan altaïen se comporte comme un animal en rut. Il introduit son phallus entre les jambes des hommes qui se trouvent dans l'obligation de mimer l'acte sexuel avec lui. Il se déplace en sautillant et chante des psalmodies. À la différence du lāto Kham Magar népalais, il porte un masque ${ }^{72}$. Chez certains groupes ethniques, c'est parfois le chamane lui-même qui assume le rôle de bouffon et qui fait rire l'assistance ${ }^{73}$. Dans ses travaux sur l'épopée et le barde au Tibet, le tibétologue Rolf Stein écrit : «Entre le bouffon et le chamane,

69. Idem, p. 78,80 .

70. Cf. par exemple Boris Chichlo, « Qui est donc Koca? », Études mongoles et sibériennes, 9, 1978, p. 7-72.

71. Evelyne Lot-Falk, « Koca-Kan, rituel érotique altaïen », Études mongoles et sibériennes, 8,1977 , p. 81 et suivantes.

72. Une vidéo postée sur Internet et filmée en pays Kham Magar (Lugum, Rukum District) en 2005 fait cependant apparaître lors de grandes cérémonies chamaniques collectives (jhānkri melā) dans l'espace villageois un clown masqué. Cf. https://www.youtube.com/watch?v=dEi3TyIHDcc\#t=1442.89234. Je remercie François Pannier d'avoir attirer mon attention sur cette vidéo.

73. À propos des chamanes iakoutes, voir F. Krauss, Das schamanentum der Jakuten, Wien, Anthropologischen Gesellschaft, 1988, cité par Anne de Sales, op. cit., p.17. Voir aussi Roberte Hamayon, La chasse à l'âme. Esquisse d'une théorie du chamanisme sibérien, Nanterre, Société d'ethnologie, 1990, p. 500, à propos des Bouriates. 
point d'opposition absolue $\gg^{74}$. Ces deux personnages auraient en commun d'opérer des passages et des transformations. Le religieux et le comique sont liés.

La grande fête agraire midje que les Yi (Nipa) du Yunnan, une minorité chinoise de langue tibéto-birmane, célèbrent en automne et qui dure sept jours révèle également les liens entre chamanisme, festivité locale et bouffonnerie. Aurélie Névot, une ethnologue, en a fait la description et l'analyse ${ }^{75}$. Cette célébration composée d'une multitude de rituels a une dimension chamanique évidente. Les chamanes bimo ouvrent les festivités, ils invoquent les ancêtres fondateurs de la localité, ils sont les maîtres de plusieurs cérémonies et ils entreprennent à cette occasion des voyages mystiques dans le cosmos. Ils président même des rituels agraires. On est frappé de les voir ainsi intervenir directement au nom de la communauté tout entière et présider aux enjeux collectifs de la localité. Ils sont à la source de ce grand rituel collectif midje qui permet à la communauté de refaire son unité et d'entrer dans une nouvelle année agricole. "Le chamane se consacre indéfiniment à la survie de son village $»^{76}$. Les aspects carnavalesques de cette fête: échanges de plaisanteries salaces, jeux et compétition, présence de bouffons qui font les pitres, sont directement liés à ce chamanisme quasi officiel, promu religion du groupe tout entier.

Les parallèles ne manquent donc pas. Mais, jusqu'à preuve du contraire, les bouffons chamaniques s'avèrent plutôt rares en Himālaya. Les Tamang, chez qui le chamanisme est fondamental bien que plus marginal au plan social que chez les Yi et les Kham Magar, pourraient faire appel à des clowns Kāmi, ces forgerons étant nombreux, on l'a dit, à vivre à proximité. Ils ne le font pas. Leur quête des âmes égarées apparaît plus «sérieuse». Leurs chamanes ont d'autres moyens d'effrayer les mauvais esprits et ne sont pas attachés aux fêtes collectives des collectivités rurales. Dans le clown grotesque, je verrai donc moins un trait fondamental

74. Rolf Stein, Recherches sur l'épopée et le barde au Tibet, op. cit., 1959, p. 392.

75. Aurélie Névot, Comme le sel, je suis le cours de l'eau. Le chamanisme à écriture des Yi du Yunnan (Chine), Nanterre, Société d'ethnologie, 2008, ch. 12, tout particulièrement p. 215

76. Idem, p. 207. La centralité du chamanisme chez les Yi évoque en tous points le rôle déterminant que jouent les chamanes Kham Magar du Népal dans la vie sociale du groupe. 
de l'institution chamanique que le signe plus général des liens fondamentaux qui unissent comique et liturgique dans nombre de cérémonies religieuses.

La présence de bouffons dans les rites collectifs est du reste connue et a été maintes fois signalée en divers points de l'Himālaya (comme ailleurs dans le monde). La photo de couverture de mon livre sur le village de Pyangaon ${ }^{77}$, où l'on voit un Kāmi, forgeron de basse caste Parbatiya, impétueux et imprévisible, danser et faire le fou devant des musiciens néwar sagement rangés en ordre de procession, lors de la fête religieuse de la localité, illustre à elle seule ce phénomène. Le burlesque et le rituel sont liés. Le Kāmi, étranger à la communauté, est le bouffon de la fête. Manifestement ivre, il interpelle tout le monde, fait voler en éclats les interdits et la bienséance. On ne le blâme pas, c'est son rôle. Même dans la vie quotidienne, les gens de hautes castes considèrent souvent les personnes de bas statut comme réfractaires à toutes règles de bienséance.

\section{LES DÉMONS PERSONNIFIÉS}

Ici et là, de par les confins himālayens, les démons sont personnifiés lors de mascarades et expulsés de la communauté ou mis à mort à date fixe. La mise en oeuvre de tels rituels apotropaïques et les personnes choisies pour tenir ces rôles présentent des aspects comiques indéniables. La culture néwar est particulièrement riche en ce domaine. Prenons le Gathāmugah, fête qui tombe le quatorzième jour de la quinzaine sombre du mois de Sāun (juillet-août). Cette festivité commémore la mort du démon cannibale Ghantakarna qui terrifiait les habitants de la vallée de Katmandou. Il détruisait récoltes et maisons, il dévorait la chair des enfants dont il se régalait. Finalement, Ghantakarna le sanguinaire fut soumis par un prêtre bouddhiste aux pouvoirs tantriques particulièrement puissants. Les paysans Jyāpu néwar l'achevèrent à coups de pierres. Ce jour du mois de Sāun, des effigies du démon sont façonnées avec de la paille de riz aux croisements de rues.

77. Gérard Toffin, Pyangaon, communauté néwar de la vallée de Kathmandou. La vie matérielle, Paris, Éditions du CNRS, 1977. 
À la place de la tête on place un van sur lequel sont dessinés les yeux, une bouche, un nez. Il s'agit d'un véritable masque plat représentant le démon. L'effigie a un sexe rouge dressé. À la paille, on accroche des poupées en tissu de couleur attrayante. Séduits par ces jolies figurines, les mauvais esprits (surtout ceux qui s'attaquent aux jeunes enfants) s'y glissent. La poupée, jeu des petites filles néwar, sert d'intermédiaire entre le monde des humains et celui des esprits. Le ludique une nouvelle fois se voit investi de connotations religieuses, comme dans les rituels chamaniques sibériens ${ }^{78}$. Le soir venu, ces structures de paille sont battues et brûlées. Puis les jeunes du village (la coutume est aussi observée dans les villes) vont les jeter en courant dans la rivière voisine. Cette épuration se fait dans la joie, les rires et la bonne humeur. Elle peut toutefois devenir violente car le rite réveille souvent de vieilles rivalités entre quartiers voisins de la localité.

Un trait retient particulièrement l'attention. Le visage peint en rouge, le corps vêtu de haillons, un jeune homme Pode, appartenant à une caste de pêcheurs-balayeurs de très bas statut, doit faire mine de s'asseoir sur l'effigie maléfique avant qu'on y mette le feu. Parfois, c'est ce bouffon d'un soir qui allume luimême la paille. Ailleurs, c'est lui qui doit accrocher les poupées à l'éphémère représentation du démon. Il est conspué, accablé d'injures et d'obscénités, comme s'il incarnait lui-même le démon que l'on met symboliquement à mort ${ }^{79}$. Le rire cache ici un rite prophylactique, démonifuge. En figurant de manière cocasse les démons, les gens les contrefont et les ridiculisent. Ils les mettent à distance et finissent par détruire la peur que ces êtres malfaisants inspirent.

La fête de Gathāmugah achevée, la saison des danses lākhay commence. En néwari le terme lākhay désigne un démon que des jeunes gens incarnent tous les ans à la même période (de fin juillet à septembre) en dansant dans les rues des agglomérations. Le mot lākhay vient du sanskrit rakșas qui désigne les démons cannibales. Ces ogres appartiennent donc à la même catégorie que le croquemitaine précédent, Ghantakarna. La tête des danseurs

78. Cf. Charles Stépanoff, Chamanisme, rituel et cognition. Chez les Touvas de Sibérie du Sud, Paris, Éditions de la MSH, 2014, p. 363-365.

79. Gérard Toffin, Société et religion chez les Néwar du Népal, Paris, Éditions du CNRS, 1984, p. 518-519. 
est couverte d'un large masque de couleur rouge surmonté d'une énorme crinière hirsute de poils de yak. Les yeux du démon sont très rapprochés, parfois ils biglent. Les canines protubérantes laissent peu de doute sur les intentions féroces du démon. Les jeunes danseurs sont vêtus d'une chemise de brocard et d'une jupe de couleur qui leur arrive à mi-mollet. Ils ont une lourde ceinture de grelots autour de la taille qui tintent lorsqu'ils se déplacent. Le lākhay (qui se produit généralement seul ou par paire) sautille et virevolte sur lui-même pieds nus au rythme d'un petit orchestre de tambourinaires et de cymbaliers. Il est parfois accompagné d'un ou deux autres personnages comiques, plus petits que lui, joués par des enfants. Eux aussi sont masqués. Dans certains cas, ils asticotent les lākhay avec des bâtons. Dans d'autre cas, ils figurent la femme ou l'ami du démon et esquissent des pas de danse avec lui.

Ces mascarades sont plus spécifiquement liées aux agriculteurs néwar Jyāpu et quelques autres castes associées, tels les potiers Kumāh. Très populaires, elles se déroulent dans un climat de joie et d'allégresse. Tout le monde se jette dans la rue, enfants, hommes et femmes, pour profiter du spectacle et suivre pas à pas le groupe dansant. Le ou les interprètes quémandent souvent de l'argent au public pour couvrir les frais des repas de fête du groupe qui a organisé l'évènement. De temps à autre, le lākhay fonce dans la foule créant un mouvement de panique, moitié amusé, moitié craintif. Ces moments d'interaction sont particulièrement appréciés des spectateurs. Les saynètes atteignent leur point culminant (lākhay jātrāa) le jour de la fête de la vache, Sā Pāru, le premier jour du mois lunaire de Bhādau, quinzaine sombre (août - septembre), et se terminent en principe huit jours plus tard lors de la commémoration de la naissance du dieu Kṛ̣ṇa qui marque le triomphe des forces divines sur les démons et anti-dieux asura. Certains lākhay peuvent cependant se produire jusqu'à la fin de ce mois, notamment à l'occasion de la fête d'Indra. Après quoi le désordre cosmique de la saison des pluies s'achève et ces démons disparaissent jusqu'à l'année suivante ${ }^{80}$.

80. Sur les aspects religieux du calendrier des fêtes néwar, tout particulièrement durant la saison des pluies, voir G. Toffin « Les Quatre mois dans le calendrier des fêtes de la vallée de Katmandou, Népal », De l'Arabie à l'Himalaya. Chemins 
En émigrant dans les bazars des collines népalaises, à l'est comme à l'ouest, pour s'établir comme commerçants dans de minuscules échoppes alignées le long de rues de terre battue, les Néwar ont exporté avec eux ces danses lākhay. On peut en observer de nos jours non seulement dans la vallée de Katmandou, mais aussi à Pokhara, Dhankuta, Jumla, Okhaldhunga, Bhairahawa et ailleurs. Dans biens des cas, les Néwar de cette diaspora ont perdu nombre de leurs coutumes anciennes, mais ils ont conservé leur fête de läkhay. La fête prend des aspects particulièrement spectaculaires à Jumla dans l'extrême ouest du Népal. Jusqu'il y a peu de temps encore, de nombreux figurants grimés et habillés de costumes pailletés représentant les héros de la geste du Rāmāyaṇa (Rāma, Lakṣmaṇa, Kṛ̣ṇa, Balarāma) y participaient à côté du lākhay local et de ses acolytes ${ }^{81}$. Ils finissaient par mettre à mort le démon - de manière symbolique s'entend. Ces liens entre démons incarnés et héros du Rāmāyaṇa existent aussi dans la vallée de Katmandou. Ils accentuent la dimension religieuse, épique en l'occurrence, de telles cérémonies comiques.

Ce personnage démoniaque a tellement marqué les imaginations que plusieurs groupes de populations non néwar l'ont ici et là adopté. L'emprunt s'est fait moyennant quelques modifications et l'incorporation de traits culturels endogènes ${ }^{82}$. Lorsque de telles mascarades de lākhay sont organisées par des gens de culture Parbatiya, ce sont souvent des castes de bas statut, Damāī et Badī, qui interprètent le démon néwar ou ses comparses, un trait asymétrique que nous avons relevé plus haut. Dans les collines, ces mascarades incluent de nombreux clowns et bouffons (souvent appelés swāng en népali), masqués ou grimés ${ }^{83}$.

D'autres groupes ethniques népalais font jouer à des clowns le rôle de mauvais esprits et de fantômes lors des cérémonies religieuses. C'est le cas en particulier des Gurung du massif des

croisés en hommage à Marc Gaborieau. Sous la direction de Véronique Bouillier et de Catherine Servan-Schreiber, Maisonneuve-Larose, Paris, 2004, p. 125-143.

81. Cf. Barry \& Lila Bishop, "Karnali, Roadless World of Western Nepal", National Geographic, 140, 5, 1971, p. 656-688.

82. Sur ce sujet, voir le chapitre très documenté du livre Mascarades de l'Himalaya, op. cit., p. 150-160.

83. En Inde du nord, le mot svāng ou sāng désigne une forme de théâtre populaire, dansé et accompagné de musique, proche du théâtre nautankī. Il comprend nombre de duos comiques. Le mot népali vient probablement de là. 
Annapurna. Lors des secondes funérailles pae (ou pae lava, pai) célébrées théoriquement 49 jours après un décès (mais souvent beaucoup plus tard), les membres de ce groupe mettent en scène des pantomimes dansées opposant prêtres tribaux klihbri (klevri) et esprits malfaisants ${ }^{84}$. Ces derniers, incarnés par les gendres de la famille ( $m o h$ ou $m a$ ), sont masqués. Ils ont la tête coiffée d'herbes sauvages et sont vêtus de vêtements colorés. Ils s'exhibent en sautillant, font des gestes désordonnés, souvent scatologiques, et courent dans tous les sens, parfois des pénis factices à la main. Ces mauvais esprits (mankhu en gurung) sont là pour empêcher les âmes des défunts de parvenir au pays des morts et de devenir des mânes bienveillants. Ils cherchent à capturer l'âme des personnes décédées et à se saisir de l'effigie mortuaire, plah, que les parents du défunt fabriquent à cette occasion (avec des tissus fournis par les «oncles maternels ») pour représenter le trépassé. Leurs gestes saccadés s'opposent en touts points aux pas réglés et harmonieux des prêtres tribaux klihbri qui dansent accompagnés de leurs tambours et de leurs cymbales et qui portent sur la tête les images des cinq Buddhas. Les klihbri ont pour fonction de mener l'âme du défunt dans le pays des ancêtres. Eux seuls connaissent la route (Pignède, 1966, p. 346). Les gendres aux accoutrements clownesques cherchent à les retenir.

Des faits similaires ont été rapportés par Brigitte Steinmann, à propos des secondes funérailles (dge-ba, gewa) des Tamang de l'Est, en particulier lors des danses masquées de lamas du deuxième jour de la cérémonie ${ }^{85}$. Significativement, au sein de ce groupe ethnique, ce sont aussi des gendres, choisis parmi les

84. Sur ce sujet, voir les films de Alan Macfarlane postés sur le site de l'université de Cambridge en Angleterre ainsi que les commentaires de cet anthropologue, spécialiste des Gurung, The Spirit Word of the Gurungs, notes to accompany over fifty films made between 1968 and 2010 in central Nepal (with Sarah Harrison), pp. 150-162 (https://sms.cam.ac.uk/collection/1640057 https:// sms.cam.ac.uk/search ? qt_type $=$ sms\&qt $=$ gurung $+\& \mathrm{x}=0 \& y=0$ ). Sur les rituels funéraires gurung, voir aussi l'étude fondamentale de Bernard Pignède, Les Gurung, une population himalayenne du Népal, Paris/La Haye, Mouton, 1966, ainsi que l'étude de Stan Royal Mumford, Himalayan Dialogue. Tibetan Lamas and Gurung Shamans in Nepal, Madison, The University of Wisconsin Press, 1989 sur les Gurung de Gyasumdo (région de Manang).

85. Voir Brigitte Steinmann, «La cérémonie funéraire chez les Tamang de l'Est », Bulletin de l'École française d'Extrême-Orient, 1987, tome LXXXVI, p. $250-252$. 
lamas, qui jouent souvent le rôle de clowns et qui portent des simulacres de phallus.

Chez les Gurung comme chez les Tamang (et peut-être parmi d'autres groupes ethniques népalais), la cérémonie funéraire se transforme donc en une bataille rituelle qui n'est pas sans rappeler les combats que miment les chamanes contre les entités invisibles. Dans ces saynètes comiques se trouve comme condensé le système social de ces deux ethnies, lequel est basé sur le mariage de cousins croisés et l'opposition entre preneurs et donneurs de femme. Dans ce système de parenté, les gendres sont tenus à toute une série d'obligations envers leurs beaux-parents et leurs oncles maternels ${ }^{86}$. Ils ont un statut inférieur de débiteur à l'endroit de leur belle-famille. Ces liens asymétriques sont comme rejoués lors des cérémonies funéraires pae. Le sérieux et le comique apparaissent ici ancrés dans les réalités sociales et les relations de parenté.

\section{CONCLUSion}

Des clowneries s'immiscent donc fréquemment dans les rituels et les cérémonies des populations de l'Himālaya; le religieux se conjugue alors sur le mode comique. Il ne s'agit pas seulement, on l'a vu, de divertissement, mais aussi de sacré, un sacré volatile fait de mouvement et d'audace. Les bouffons en question incarnent des forces surnaturelles. Comme les chamanes et certains spécialistes rituels, ils transportent les spectateurs dans un autre monde, atemporel, au-delà des règles coutumières. Les scènes qu'ils représentent, les parades, les costumes bariolés, toute cette effervescence, les cavalcades, la multiplicité soudaine évoquent des univers imaginaires dans lesquels évoluent les intercesseurs, les fous inspirés, les démons. Ignorant les obstacles, les clowns rituels traversent d'un pas léger les frontières habituellement étanches. Comment s'opposer à eux et les réprimander? Nous ne sommes plus dans une quelconque facette himālayenne du rire, mais devant un trait général du comique des sociétés non modernes. La figure du retournement, de l'inversion (le même devient l'autre), celle-là même que Georges Bataille a mis en lumière dans son œuvre, est

86. À ce propos, voir l'analyse de Bernard Pignède, 1966, p. 276-277. 
ici essentielle. Le sacré sévère se transforme en sacré sacrilège, celui que Bataille, justement, qualifie de « sacré gauche».

Si des bouffons s'exhibent, c'est, bien sûr, "pour rire » et «faire rire », pour contribuer à l'allégresse générale de la fête et des cérémonies religieuses par des gestes obscènes, libres de toute entrave, selon un schéma presque toujours identique. Cette confusion générale des identités et des statuts est plus proche de l'opéra bouffe que de la satire et de la comédie des mœurs. Elle crée un air de fête et de carnaval, sans souffrance, même si le négatif imprègne fortement les représentations démoniaques. Le clown, tel un personnage de théâtre, se caractérise par des facultés de dédoublement; son être excentrique se démultiplie et se métamorphose. Cette capacité le situe au-delà des attaches terrestres. Elle cache un arrière-monde invisible fait d'ambiguïté, de rituels de fertilité, de hiérogamie entre ciel et terre et de lutte contre les démons. La bouffonnerie n'est triviale qu'en apparence.

Les fonctions religieuses de tels spectacles ludiques peuvent être regroupées sous trois grandes catégories. $1^{\circ}$ ) Il s'agit souvent d'attirer les mauvais esprits, de façon à les capturer, à distraire leur attention, leur faire peur, les repousser, les caricaturer, les figurer sous des aspects grotesques pour mieux s'en débarrasser. Parfois, c'est l'esprit malfaisant lui-même que ces clowns incarnent. $2^{\circ}$ ) Ces scènes comiques contribuent aussi à réjouir les dieux, à leur plaire : c'est un spectacle qu'on leur offre et dont on attend, en retour, des bénéfices. $3^{\circ}$ ) Les mascarades enfin concourent aux rituels de fertilité (simulacre d'accouplement, obscénités, grivoiseries) et de refondation symbolique des communautés consécutive à un moment de désordre intense. Le grand tourbillon accouche d'une vie nouvelle. D'où la fréquente association des clowns rituels avec le passage d'une saison à l'autre ${ }^{87}$. La bouffonnerie est ici conjonctive, comme le rite selon Lévi-Strauss : elle contribue à générer un ordre nouveau.

La mort appelle le rire, les diablotins farfelus se donnent en spectacle, les chèvre-pieds menacent les lamas. Ceci étant, les aspects sociologiques doivent, à chaque fois, être considérés. C'est généralement à des personnes de basses castes ou de statut dégradé ou inférieur que l'on confie les rôles de bouffons. Mais dans

87. D. Handelman, op. cit., p. 329. 
d'autres cas, ce sont des personnages religieux les plus respectés qui sont parodiés et moqués. Ici le comique reflète fidèlement les hiérarchies internes, là il inverse les positions. La plasticité des spectacles est extrême. Quant aux femmes, on les choisit rarement pour incarner ces rôles. Ce sont des hommes travestis qui les représentent. Le comique et le jeu s'inscrivent dans un vaste système de différences sociales qu'il appartiendra à d'autres études plus approfondies de préciser.

Un autre élément retient l'attention. Le comique ne peut s'analyser uniquement en termes de culture populaire. Contrairement aux thèses dichotomiques avancées par Bakhtine dans son analyse du comique occidental médiéval, des conceptions religieuses, telles celle du "saint fou », irriguent le rire himālayen. Culture savante et culture populaire se mêlent. Les bouffons atsara tibétains et vidūṣaka indiens ne se comprennent qu'à la lumière de notions anciennes. Ils appartiennent à la vieille littérature sanskrite autant qu'aux traditions populaires contemporaines. Les deux volets méritent d'être étudiés de concert. Autre exemple: les thèmes du travestissement, de la duperie sont légion dans l'épopée canonique du Mahābhārata (Arjuna par exemple qui se déguise en eunuque dans le livre IV). Ce sont des ingrédients narratifs de base.

Le geste, les positions, les postures de ce comique sacré reposent sur un petit nombre de figures toujours identiques. La grivoiserie, la scatologie, le travestissement en dieux, yogīs ou femme y sont toujours de mise. Une grammaire de ce comique gestuel mériterait d'être élaborée. Ce pourrait être le sujet d'une autre recherche et la matière à de futurs développements. Parfois, ce comique purement muet s'articule à une parole : épopée récitée et chantée, dialogues de farce théâtrale, chants et récits divers. Dans cette rupture du temps routinier, la satire des puissants et des personnes haut placées, les sketches relatifs aux faiseurs de tours, aux ruses et aux duperies prennent alors l'ascendant.

gtoffin@vjf.cnrs.fr 